\title{
Alineación Láser para Experimentación con Fotografía Digital de Speckle (DSP)
}

\section{Laser Alignment for Experimentation with Digital Speckle Photography (DSP).}

\author{
LÓPEZ-ÁLVAREZ, Yadira Fabiola'†*, RODRIGUEZ-FRANCO, Martín Eduardo, JARA-RUIZ, \\ Ricardo y DELGADO-GUERRERO, Sergio \\ Universidad Tecnológica del Norte de Aguascalientes, Aguascalientes, Estación Rincón, Rincón de Romos, Aguascalientes, \\ 20400 México \\ 'Universidad de Guadalajara, Lagos de Moreno, Jalisco, 47460, México
}

ID $1^{\mathrm{er}}$ Autor: Yadira Fabiola, López-Álvarez/ ORC ID: 0000-0002-9041-1908, Researcher ID Thomson: T-1555-2018, CVU CONACYT ID: 375952

ID $1^{\text {er }}$ Coautor: Martín Eduardo, Rodriguez-Franco/ ORC ID: 0000-0002-6804-4777, Researcher ID Thomson: T-15392018, CVU CONACYT ID: 660892.

ID $2^{\text {do }}$ Coautor: Ricardo, Jara-Ruiz/ ORC ID: 0000-0001-7725-4138, Researcher ID Thomson: T-1532-2018, CVU CONACYT ID: 630276

ID $3^{\text {er }}$ Coautor: Sergio, Delgado-Guerrero/ORC ID: 0000-0003-2521-5887, Researcher ID Thomson: V-1747-2018, CVU CONACYT ID: 240475

DOI: $10.35429 / J O T I .2019 .9 .3 .21 .28$

Recibido 16 de Enero, 2019; Aceptado 19 de Marzo, 2019

\section{Resumen}

Las técnicas de no contacto han vuelto a la metrología óptica una de las ramas de la ciencia con mayor auge, haciendo de la Fotografía Digital de Speckle (DSP) una de las más utilizadas en la medición de cambios estructurales en superficies. Esta técnica utiliza como sistema de medición un láser y compara los cambios del patrón de speckle que se obtienen al irradiar la superficie que es sometida a deformaciones; sin embargo, la alineación de estos sistemas juega un papel importante en los resultados obtenidos. En este trabajo, se estiman los errores de alineación de un sistema óptico basado en el análisis del patrón de difracción de Fraunhofer, así como las propiedades estadísticas del patrón de speckle para campo cercano y lejano; los resultados indican que la alineación del haz de luz puede ser determinada mediante un sistema de cuantificación de franjas al pasar por una apertura circular y que el patrón de speckle altamente desarrollado tiene mayor semejanza con la distribución de intensidad mostrado en la literatura.

Alineamiento óptico, patrón de speckle, fotografía digital de speckle

\begin{abstract}
Resumen
Non-contact techniques have returned to optical metrology one of the most booming branches of science, making digital speckle photography (DSP) one of the most used in the measurement of structural changes in surfaces. This technique uses a laser as a measurement system and compares the changes in the speckle pattern obtained by irradiating the surface that is subjected to deformations; however, the alignment of these systems plays an important role in the results obtained. In this work, the alignment errors of an optical system are estimated based on the analysis of the Fraunhofer diffraction pattern, as well as the statistical properties of the speckle pattern for field near and far. The results indicate that the alignment of the light beam can be determine by a fringes quantification system when passing through a pinhole and that the highly developed speckle pattern is more similar to the intensity distribution shown in the literature.
\end{abstract}

Optical alignment, speckle pattern, digital speckle photography

Citación: LÓPEZ-ÁLVAREZ, Yadira Fabiola, RODRIGUEZ-FRANCO, Martín Eduardo, JARA-RUIZ, Ricardo y DELGADO-GUERRERO, Sergio. Alineación Láser para Experimentación con Fotografía Digital de Speckle (DSP). Revista de Invención Técnica 2019. 3-9:21-28 


\section{Introducción}

La radiación electromagnética se propaga en forma de dos vectores de onda, eléctrico y magnético donde la luz se puede describir como un fenómeno de onda electromagnética mediante una función escalar de posición y tiempo, $E(r, t)$ y $H(r, t)$ usando las ecuaciones de Maxwell.

$$
\begin{aligned}
& \nabla \times H=\varepsilon_{0} \frac{\partial E}{\partial t} \\
& \nabla \times E=-\mu_{0} \frac{\partial K}{\partial t} \\
& \nabla \cdot E=0 \\
& \nabla \cdot H=0
\end{aligned}
$$

Donde $\varepsilon_{0}$ y $\mu_{0}$ son la permitividad eléctrica y la permeabilidad magnética.

Para que una onda electromagnética se propague es necesario que el campo eléctrico y magnético satisfagan las ecuaciones de Maxwell y a su vez cada uno de los componentes de campo deberán satisfacer también la ecuación de onda (Fitzpatric, 2018).

$$
\begin{aligned}
& \nabla^{2} \mathrm{u}-\frac{1}{c_{0}^{2}} \frac{\partial^{2} u}{\partial t^{2}}=0 \\
& c_{0}=\frac{1}{\left(\varepsilon_{0} \mu_{0}\right)^{1 / 2}}=3 \times 10^{8} \mathrm{~m} / \mathrm{s}
\end{aligned}
$$

Cuando esta onda electromagnética que se propaga es distorsionada por un obstáculo, una pequeña apertura o una rendija que solo permite el paso de una porción del frente de onda y cuyas dimensiones son comparables con la longitud de onda, origina una desviación de la luz en su propagación rectilínea a este fenómeno se le conoce como difracción este efecto es una característica de los fenómenos ondulatorio y ocurren siempre que una onda presente una perturbación (Hech, 2008). Este fenómeno se basa en el principio de Huygens que ilustra que cada frente de onda contribuye como un conjunto para formar trenes de onda de tal modo que a ciento tiempo el frente de onda, será la envolvente de trenes de onda con la misma frecuencia y velocidad de la onda que se propaga (Hech, 2008).
Para el siglo XIX, Fresnel introdujo una serie de modificaciones al principio de Huygens añadiendo el concepto de interferencia, "cada punto sin obstrucción de un frente de onda, en un instante de tiempo determinado, sirve como frente de trenes de onda secundarios de la misma frecuencia que la onda primaria; la amplitud del campo óptico en cualquier punto más allá de la superposición de todos estos trenes de onda considerando sus amplitudes y fases relativas" (Hech, 2008). Posteriormente Kirchhoff demostró que el principio de Huygens - Fresnel era una consecuencia directa de la ecuación diferencial de onda, Ec. (5).

Si se toma una apertura arbitraria en el plano $(x, y)$, en el cual incide una onda plana monocromática, como muestra la Fig. 1.

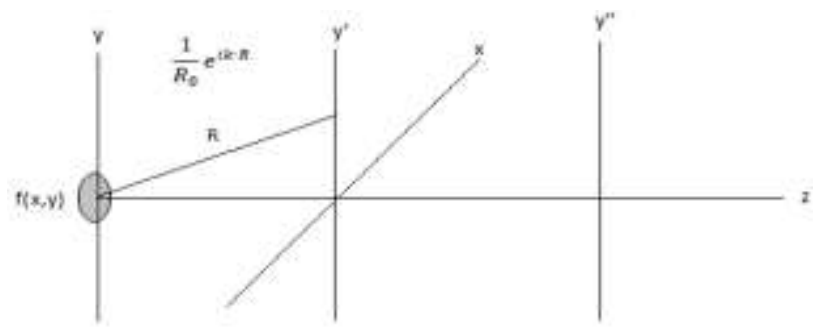

Figura 1 Diagrama de difracción de Fraunhofer de apertura arbitraria (Torres, 2010)

$\mathrm{R}$ es la distancia entre el centro de la apertura al punto de salida donde el campo es:

$g\left(x^{\prime}, y^{\prime}\right)=\frac{1}{R} \iint_{-\infty}^{\infty} f(x, y) e^{i k * R}$

Usando aproximación paraxial, es posible llegar a la transformada de Fourier que describe el efecto de la difracción de Fraunhofer de la distribución del campo.

$F(v, \mu)=\iint_{-\infty}^{\infty} f(x, y) e^{-i 2 \pi(x v+y \mu)} d x d y$

Considerando a $v$ y $\mu$ como cosenos directores

$v=\frac{x}{\lambda z}$

$\mu=\frac{y}{\lambda z}$

Pudiendo expresar a la Ec. (8) como:

$F(v, \mu)=\mathrm{A} \iint_{-\infty}^{\infty} f(x, y) e^{-i 2 \pi(x v+y \mu)} d x d y(11$ 
Donde A es una constante compleja y la Ec. (11) es llamada la integral de Fraunhofer, que puede ser usada para representar el campo en el plano de observación (Bracewell, 1965), tiene lugar en una distancia mucho más grande que el tamaño de la apertura, por lo que es llamada también difracción de campo lejano (Goodman, 2005).

La difracción de Fraunhofer con una apertura circular muestra un patrón circular en la pantalla de observación, Figura 2.

Si se considera una pantalla con aperturas geométricas simples, en las que pueden destacar las rectangulares y circulares, la aproximación al estudio de los trenes de onda producidos se realiza mediante al aproximación de Fraunhofer (Torres, 2010), se supone, entonces, una apertura circular, donde su imagen resultante corresponderá a un disco brillante rodeado de anillos, según o muestra la Fig. 2.

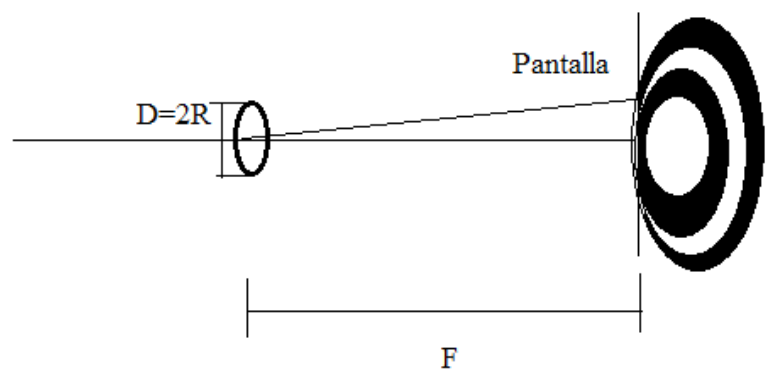

Figura 2 Diagrama de difracción de Fraunhofer de apertura circular (Molina, 2017)

Siendo $\mathrm{R}$ el radio de la apertura y $\theta$ corresponde al ángulo del primer disco obscuro, dado por la Ec. 7 y Ec. 8 .

$\frac{2 \pi R \operatorname{sen} \theta}{\lambda}=3.8317$

$\theta \approx \operatorname{sen} \theta=1.22 \frac{\lambda}{2 R}=1.22 \frac{\lambda}{D}$

Estas expresiones además de definir la geometría de la apertura, según Rayleigh, son definidas también como el ángulo mínimo entre las direcciones de incidencia de dos frentes de onda planas provenientes de dos fuentes puntuales (Hecht, 2008).

\section{Filtraje espacial y colimación.}

El filtraje espacial y su alineamiento son conocidos como el principio del desarrollo de las ciencias ópticas, consiste en una lente de corto enfoque con un pinhole (apertura en el orden de las micras) muy pequeño enfocado horizontal, vertical y longitudinalmente (Saxby,2003), cuando una lente enfoca la luz del haz, el campo eléctrico en el centro focal es la trasformada óptica del campo del objeto y está forma un patrón de difracción en este plano; en el cual, las más altas frecuencias forman manchas que están lejanas al centro óptico del patrón.

El tamaño mínimo del pinhole está dado por la Ec. (9).

$D=600 \lambda / M d$

D es el diámetro del ponhole requerido, $\mathrm{M}$, representa al magnificación, $\lambda$ la longitud de onda y $d$ el diámetro del rayo en milímetros $(\mathrm{mm})$.

Para conocer las características del pinhole así como del objetivo del microscopio a usar en el experimento es necesario conocer la magnificación del objetivo de microscopio. Existen dos maneras para la alineación de los sistemas ópticos, la primera consiste en hacer coincidir la salida del láser con la entrada del objetivo del microscopio y la segunda en alinear el pinhole con el objetivo de microscopio, otro de los puntos importantes que se beben de considerar para la alineación del sistema es la colimación de la luz, ésta consiste en que los rayos que forman el sistema de iluminación son paralelos entre sí (Hecht, 2008), ésta propiedad es la que hace posible capturar toda la luz emitida por un láser debido a que emerge de un haz de pequeño diámetro que no tiene divergencia ni convergencia.

Suponiendo a un frente de onda que se propaga en dirección $\mathrm{z}$, la localización de la cintura (waist) tanto antes como después de la distancia focal de $f$ puede encontrarse a partir de:

$\left(\frac{z \prime}{f}-1\right)=\frac{\frac{z}{f}-1}{\left(\frac{z}{f}-1\right)^{2}+\left(\frac{z_{0}}{f}\right)^{2}}$ 
Donde $z$ es la distancia desde la cintura del haz que incidirá hasta la lente, mientras que $z^{\prime}$ es la distancia respectiva desde la lente hasta la cintura del haz transmitido, $z o$ corresponde a la mitad de la profundidad del foco.

Un haz de luz colimado es un haz (típicamente un haz láser) que tiene una divergencia baja, de modo que el radio del haz no sufre cambios significativos dentro de distancias de propagación moderadas.

En conjunto el láser, objetivo de microscopio, el pinhole, la lente para colimación, un diafragma para la regulación del sistema de iluminación y la cámara CCD (Charge Coupled Device) forman parte de un sistema óptico para el desarrollo de Fotografía Digital de Speckle (DSP) en campo cercano y lejano.

\section{Fotografía Digital de Speckle (DSP)}

La metrología de moteado es una importante aportación en las técnicas de medición ópticas, utiliza la correlación de la distribución de los puntos brillantes y obscuros llamados speckle, estos patrones son formados en el espacio cuando un objeto difuso es iluminado con luz coherente (Sjoedahi, 1997), (Rastogi, 2001) dicho patrón de moteado es portador de gran información sobre la deformación de un objeto es una técnica de medición de campo completo que sirve para determinar las deformaciones (Peder, 1998).

La fotografía de moteado surgió como una alternativa a la fotografía holográfica, cuando este patrón es tomado de la superficie es posible relacionar sus cambios y asociarlos a la deformación de la muestra, la duplicación resultante del patrón puede ser observado mediante la trasformada de Fourier (Stetson, 2013).

El análisis de los patrones de speckle utilizado en DSP puede ser utilizado en campo cercano (upper) y campo lejano (lower), presentando diferencias en su contraste (Massaki, 1983).
Para realizar el análisis según DSP es necesario recurrir a herramientas estadísticas como lo son las funciones de la densidad de probabilidad, así como la función de correlación cruzada y auto correlación, dadas por las siguientes ecuaciones:

$$
\begin{aligned}
& P_{I}(I)=\frac{I}{\langle I\rangle} \exp \left(-\frac{I}{\langle I\rangle}\right) \\
& C=\frac{\sum_{k=-\alpha}^{\alpha} \sum_{l=-\alpha}^{\alpha} s_{1}(k, l) s_{2}(k+p, l+q)}{\sqrt{\sum_{k=-\alpha}^{\alpha} s_{1}(k, l)^{2} \sum_{k=-\alpha}^{\alpha} \sum_{l=-\alpha}^{\alpha} s_{2}(k, l)^{2}}}
\end{aligned}
$$

\section{Metodología}

Para el desarrollo de este experimento se utilizó un láser $\mathrm{He}-\mathrm{Ne} 632.8 \mathrm{~nm}$, un objetivo de microscopio a $20 \mathrm{x}$, un pinhole de $20 \mu \mathrm{m}$, una cámara CCD con un diafragma colocado en dicha cámara con una distancia focal de $0.5 \mathrm{~m}$ y una computadora para procesar los datos.

Se resume el procedimiento, para el desarrollo según los sistemas de las Figuras 3,4 y 5 .

- Centrar el rayo láser en el objetivo de microscopio.

- Alinear el pinhole con el objetivo de microscopio.

- Colocar una pantalla de observación.

- Instalar la cámara CCD con un diafragma, para la captura y procesamiento de datos.

- Colocar una lente biconvexa para la colimación del haz laser.

- Capturar las imágenes del patrón de speckle que es reflejado en la pantalla de observación.

- Obtener el patrón de speckle cuando el diafragma está completamente abierto y cerrado, campo cercano y lejano, Figura 8.

Los arreglos ópticos que se utilizaran serán los que se muestran a continuación. 


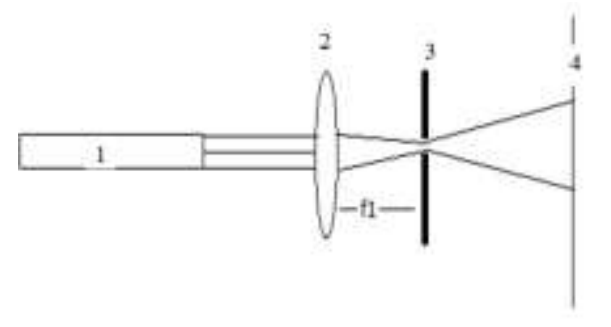

Figura 3 Sistema óptico para filtraje espacial, (1) láser HeNe, (2) Objetivo de microscopio, (3) Pinhole (apertura micrométrica) y (4) Pantalla de observación

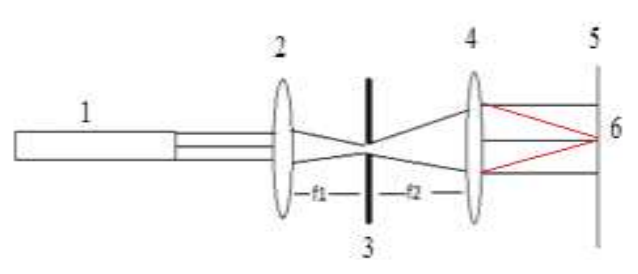

Figura 4 Sistema óptico para filtraje espacial y luz colimada, (1) Láser He- Ne, (2) Objetivo de microscopio, (3) Pinhole (apertura micrométrica), (4) Lente biconvexa, (5) Pantalla de observación, (6) Luz colimada, f1 y f2 son las distancias focales para el experimento

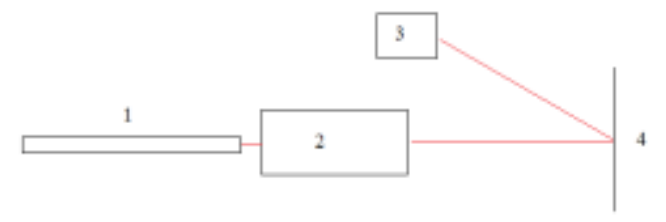

Figura 5 Sistema óptico para obtener los patrones de speckle en campo cercano y lejano, (1) Láser He- Ne, (2) Sistema Figura 3, (3) CCD, (4) Pantalla de observación

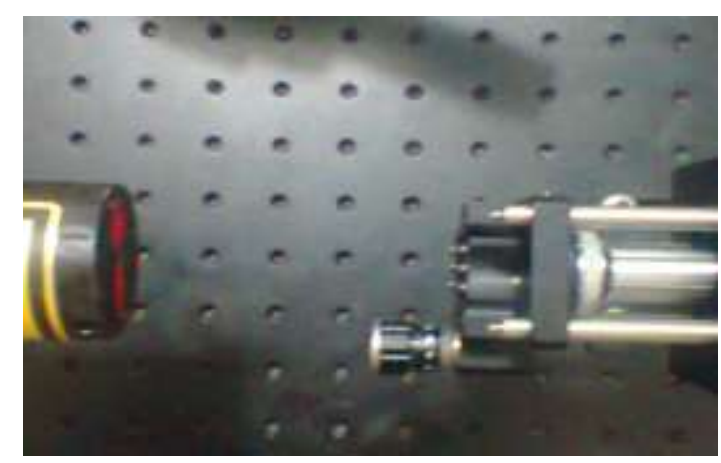

Figura 6 Filtraje espacial, laser, objetivo de microscopio $\mathrm{y}$ pinhole, con movimiento en los tres ejes

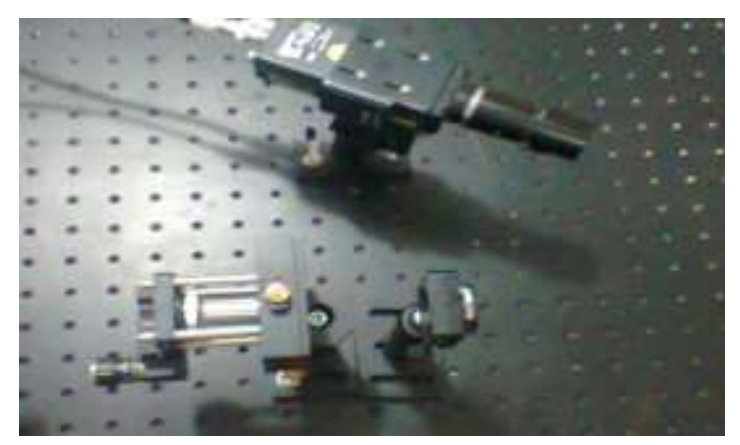

Figura 7 Sistema para obtener patrones de speckle en campo cercano y lejano

\section{Resultados}

La Figura 8 muestra el haz de luz obtenido después de realizar la colimación, en ella se puede ver que se presentan franjas de interferencia producidas por la presencia de un obstáculo en la dirección de propagación del frente de onda, para el caso de (b, dichas franjas desaparecieron, lo que indica que el haz de luz láser está más próximo al centro del pinhole, para el caso del (c, tanto las franjas de interferencia como los puntos del patrón de speckle se presentan más tenues, lo que indica que la luz láser está alineada al pinhole.
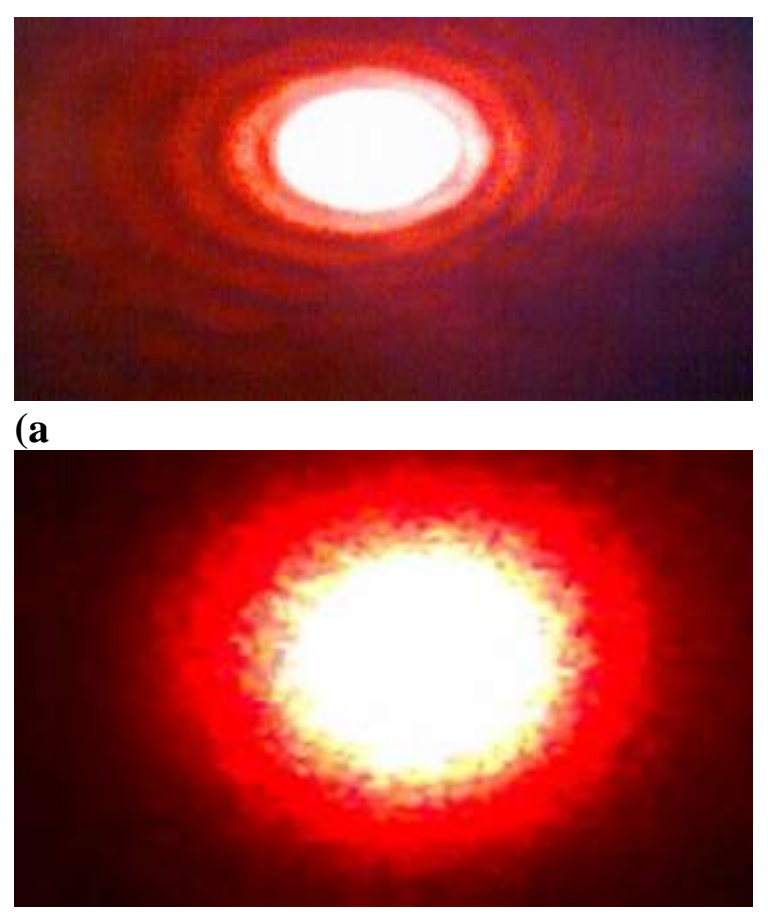

(b

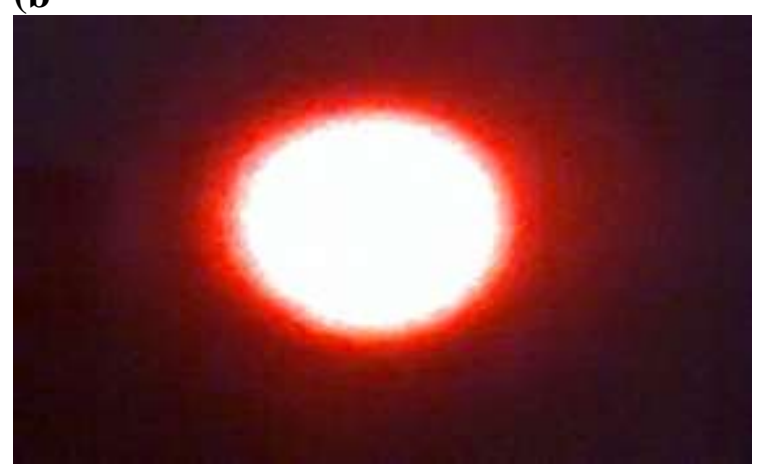

(c

Figura 8 Patrón de Difracción de Fraunhofer con una apertura circular (a Sin alinear láser, (b Láser con alineación mostrando patrones de speckle, (c Láser alineado al objetivo del microscopio.
LÓPEZ-ÁLVAREZ, Yadira Fabiola, RODRIGUEZ-FRANCO, Martín Eduardo, JARA-RUIZ, Ricardo y DELGADO-GUERRERO, Sergio. Alineación Láser para Experimentación con Fotografía Digital de Speckle (DSP). Revista de Invención Técnica 2019 
Para obtener el patrón de speckle se utilizó el sistema de las Figuras 5 y 7, los resultados se muestran en la Figura 9, para este caso el diafragma se colocó en la entrada de la cámara CCD abriéndolo por completo, se puede ver que el área de estudio es más iluminada y los patrones de speckle están poco definidos.

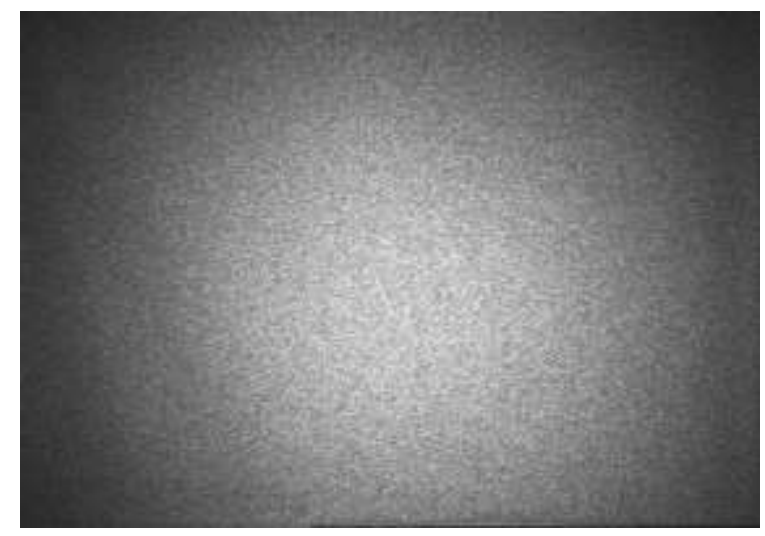

Figura 9 Patrón de moteado con diafragma totalmente abierto, menor profundidad de campo

Para el caso de la Figura 10, el patrón de speckle se obtuvo cerrando completamente el diafragma, en ella se puede observar que la distribución de la intensidad presenta mayor rugosidad, y que los patrones de speckle son más visibles.

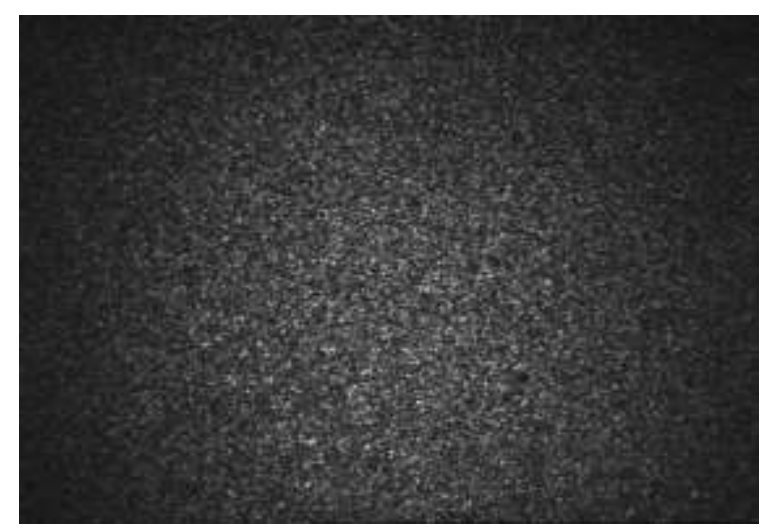

Figura 10 Patrón de moteado con diafragma cerrado, mayor profundidad de campo.

Se realizó el análisis de la función de densidad de probabilidad y del comportamiento de los coeficientes de correlación para diferentes aperturas del diafragma.
La Figura 11 muestra la función de densidad de probabilidad normalizada para campo lejano, según la ecuación (16), se puede apreciar que esta distribución esta pronunciada hacia las máximas intensidades de estudio; sin embargo para el caso de la función de densidad de probabilidad para campo cercano, Figura (13), se muestra un corrimiento hacia las mínimas intensidades, este comportamiento del sistema es debido a la cantidad de iluminación que el diafragma deja entrar al sistema de adquisición.

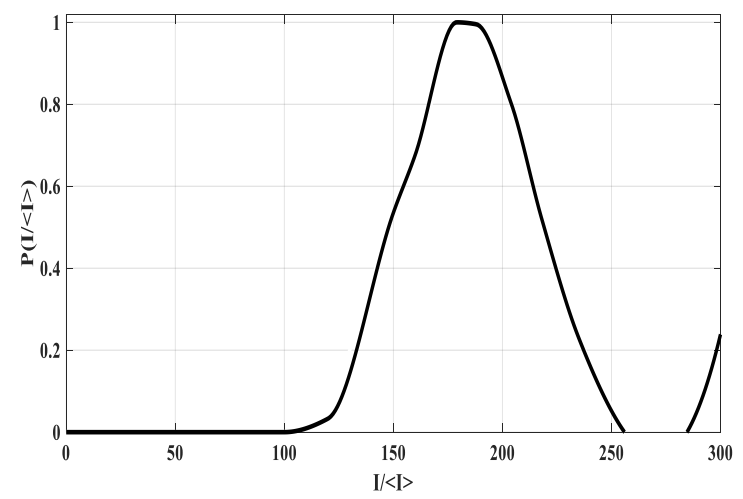

Figura 11 Función de la densidad de probabilidad del patrón de speckle, menor profundidad de campo

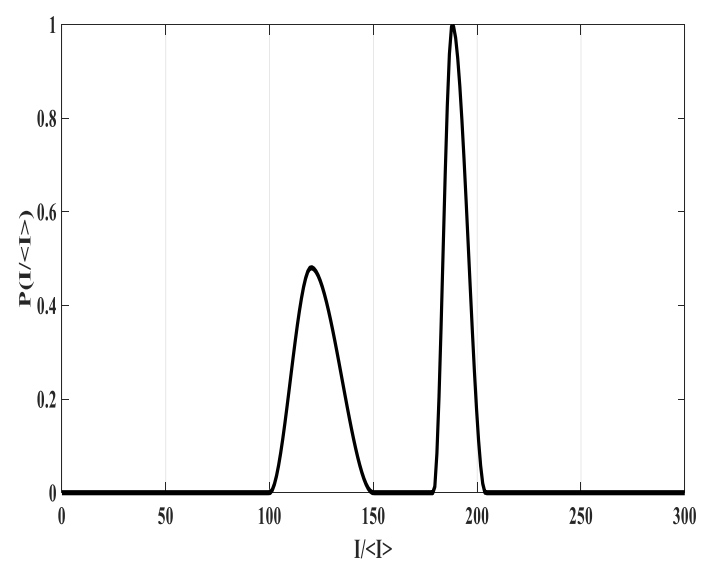

$(\mathrm{a}$

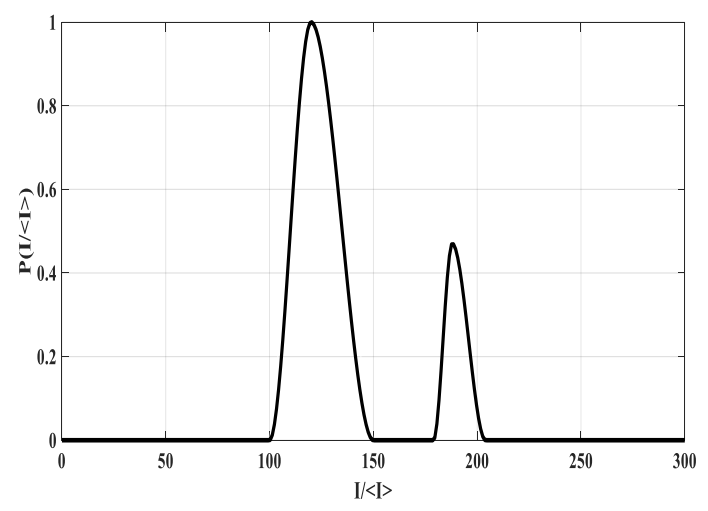

(b

Figura 12 Función de la densidad de probabilidad del patrón de speckle para campo lejano, cerrando el diafragma a razón de $f / 2$, hacia mayor profundidad de campo 
La Figura 12 muestra el comportamiento de la función de densidad de probabilidad para el patrón de speckle a medida que se acerca al rango de trabajo de un speckle desarrollado obtenido con el diafragma cerrado.

Para el caso de la Figura 13, esta es considerado como la representación adecuada de la función de densidad de probabilidad, según la literatura (Henao, 1997), aunque las dos funciones presentan una forma exponencial negativa, se recomienda en este caso trabajar en campo cercano.

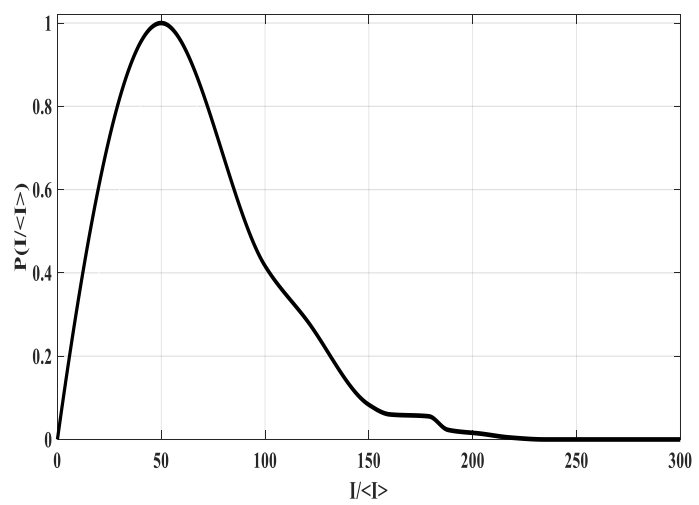

Figura 13 Función de la densidad de probabilidad del patrón de speckle para mayor profundidad de campo

\section{Conclusión}

Se dan a conocer las técnicas y proceso para realizar la alineación de luz láser usado en un sistema de pruebas en metrología óptica, mediante el reconocimiento de franjas originadas por la presencia de un obstáculo, en específico, la apertura circular, el sistema muestra reducción de dichas franjas a medida que el frente de onda se alinea con el pinhole.

Se calcularon las funciones de densidad de probabilidad de los patrones de speckle que se presentan antes de la medición de la deformación usando DSP, concluyendo que el más apto para trabajar con técnicas ópticas es el obtenido con el diafragma cerrado y mayor profundidad de campo, esto debido a sus características de distribución y forma exponencial negativa.

Se muestra, por lo tanto el procedimiento anterior a la técnica óptica por desarrollar, reduciendo los errores en los cálculos de las propiedades de los patrones de speckle utilizados en DSP y otras técnicas ópticas.
Sugiriendo entonces realizar una correcta alineación en el sistema óptico a utilizar así como el uso del patrón de speckle con mayor profundidad de campo.

\section{Referencias}

Bertolano H., Vettorel S. (2017), Fisica: Ondas Electromagneticas, Instituto Politecnico, Universidad del Rosario.

Bracewell R. N. (1965), The Fourier transform and its applications. New York: McGraw-Hill.

Fitzpatric R. (2008), Maxwell' Equations and the Principles Electromagnetism, United States of America: Infinity Science Press LLC.

Goodman J.W. (2005), Introduction to Fourier Optics, United States of America, Roberts\&Company Publishers.

Hecht E. (2008). Óptica, Madrid (España), Pearson Educación.

Henao R.J. H., (1997), Tesis de Doctorado: Estudio de Técnicas Speckle Opto-Digitales, Colombia.

Johnson P. (1998), Dual-beam Digital Speckle Photography, Strain Field Measurements in Aerospace Applications, Luleå University ofTechnology, Luleå, Sweden.

Massaki I. (1983), Statical Propieties of Optical Fiber Speckle, Hokkaido University, pp 89.

Molina C. D., Calvo A. F., Pamplona L. E., (2017), Desarrollo de un módulo experimental de difracción de la luz para un laboratorio de física controlado de forma remota, págs. 100111.

Rastogi P.K. (2001), Digital Speckle Interferometry and Related Techniques, John Wiley \&Sons Ltd, England.

Saxby G. (2003), Practical Holography, Boca Raton, FL, CRC Press.

Sjoedahi M., Synnergren P., Johnson P. (1997), Applications of digital speckle phoography in experimental mechanics, PIE 3098, Optical Inspection and Micromeasurement II. 
Stetson K. (2011), Experimental Mechanics , Analysis of speckle photographs by subtracting phase functions of digital Fourier transforms, 53 1799.

Torres, C. O.; Mattos, L.; Jiménez, C.; Castillo, J.; Torres, Y., (2010), Transformada fraccional de Fourier en el caso de un plano imagen inclinado, Ingeniería y Ciencia, pp. 51-64. 